\title{
Two Heterogeneous Servers Limited Capacity Markovian Queueing System Subjected to Varying Catastrophic Intensity
}

\author{
Gulab Singh Bura \\ Dept. of Mathematics \& Statistics, \\ Banasthali University, Rajasthan
}

\begin{abstract}
In this paper we consider a limited capacity Markovian queueing system with two heterogeneous servers subjected to varying catastrophic intensity. The transient solution of the model has been obtained and various measures of performance have been computed numerically with the help of simulation technique. The steady state solution of the system has also been provided.
\end{abstract}

\section{Keywords}

Transient Solution, Varying Catastrophic Intensity, Simulation, Markovian Queueing System, Steady State Solution

\section{INTRODUCTION}

The single server Markovian queueing system has been the object of systematic and through investigation for a long time. In real life it is not necessary that a queueing system should have only one server. Practically they may have more than one server identical or non-identical in their functioning. Heterogeneity of service is a common feature of many real multi server queueing situations. The heterogeneous service mechanism allows the customers to receive different types of services. Queueing model with heterogeneous server were rarely treated in research. A Markovian queueing system with balking and two heterogeneous servers has been discussed by Singh [1970]. In recent year the attention has been focused on queueing model with catastrophes. A large number of research papers are available for queueing model under the influence of catastrophe see. e.g. Chao (1995), Kumar and Arivudainambi (2000), Jain and Kumar (2005a, b, c; 2006), [Crescenzo et al. (2003), and Jain and Kanethia (2006)], Brockwell et al., (1982) and Bartoszynski et al., (1989)]. Recently, the queueing system with two heterogeneous servers subject to catastrophes has been discussed by Kumar, B.K., Madheswari, Pavai S. and Venkatakrishnan, K.S., (2007). In the above mentioned work, all the researchers' have used the assumption that the occurrence of catastrophe destroys all the customers in a queuing system. But it is not always the case. So necessary amendment is incorporated in the paper of Jain and Bura [2010] in the form of varying catastrophic intensity to destroy a finite number of customers at a time. As a result of varying intensity of catastrophe the number of customers destroyed instantaneously on the occurrence of a catastrophe varies between 1 and $\mathrm{N}$ not necessary all in the queueing system. Although result has been reported on queueing models with two heterogeneous servers subjected to catastrophes, but no work has been found in the literature which studies queueing model with two heterogeneous servers subjected to varying catastrophic intensity. The catastrophic intensity may follow any appropriate distribution but in this paper we have considered discrete uniform distribution and modified binomial distribution. The concept of varying catastrophic intensity has numerous applications in a wide variety of areas such as computer communications, agriculture and biosciences etc.

\section{QUEUEING MODEL}

The queueing model investigated in this chapter is based on the following assumptions:-

(i) The customers arrive in the system one by one in accordance with a Poisson Process in a single queue with rate $\lambda>0$.

(ii) There are two servers: server 1 and server 2. The service times of the customers are independently identically exponentially distributed with rates $\mu_{1}$ and $\mu_{2}$ respectively.

(iii) A customer who arrives, when there are zero customers in the system, joins the server 1 with probability $\mathrm{p}$ and the server 2 with probability $1-\mathrm{p}$.

(iv) The queue discipline is first- come- first- served.

(v) Initially, there are zero customers in the system.

(vi) When the system is not empty, catastrophes occur according to a Poisson process with rate $\xi$ and intensity $\mathrm{C}_{\mathrm{r}}$

$$
(\mathrm{r}=1,2,3, \ldots ., \mathrm{N}), \sum_{\mathrm{r}=1}^{\mathrm{N}} \mathrm{C}_{\mathrm{r}}=1 \text { It depends upon }
$$

the intensity of the catastrophe that how many customers are destroyed instantaneously.

(vii) The capacity of the system is limited to N. i.e., if at any instant there are $\mathrm{N}$ customers in the system, then the customers arriving in the duration for which the system remains in state $\mathrm{N}$ are not permitted to join the queue and considered lost for the system with probability one.

(viii) In case the catastrophe leaves only one customer in the system then the probability of his being served by server 1 is $p$ and that of server 2 is $(1-p)$. 
Define

$P_{n}(t)=$ The probability that there are $n$ customers in the system at time $t$.

$\mathrm{P}_{1,0}(\mathrm{t})=$ The probability that there is one customer in the system and he is being served by the server 1 .

$\mathrm{P}_{0,1}(\mathrm{t})=$ The probability that there is one customer in the system and he is being served by the server 2 .

The differential- difference equations governing the system are:

$$
\begin{aligned}
& \mathrm{P}_{0}^{\prime}(\mathrm{t})=-\lambda \mathrm{P}_{0}(\mathrm{t})+\mu_{1} \mathrm{P}_{1,0}(\mathrm{t})+\mu_{2} \mathrm{P}_{0,1}(\mathrm{t})+\xi \sum_{\mathrm{n}=1}^{\mathrm{N}} \sum_{\mathrm{r}=\mathrm{n}}^{\mathrm{N}} \mathrm{c}_{\mathrm{r}} \mathrm{P}_{\mathrm{n}}(\mathrm{t}) \\
& \mathrm{P}_{1,0}^{\prime}(\mathrm{t})=-\left(\lambda+\mu_{1}+\xi\right) \mathrm{P}_{1,0}(\mathrm{t})+\lambda \mathrm{p} \mathrm{P}_{0}(\mathrm{t})+\mu_{2} \mathrm{P}_{2}(\mathrm{t})+\xi \mathrm{p} \sum_{\mathrm{r}=1}^{\mathrm{N}-1} \mathrm{c}_{\mathrm{r}} \mathrm{P}_{(1+\mathrm{r})}(\mathrm{t}) \\
& \mathrm{P}_{0,1}^{\prime}(\mathrm{t})=-\left(\lambda+\mu_{2}+\xi\right) \mathrm{P}_{0,1}(\mathrm{t})+\lambda(1-\mathrm{p}) \mathrm{P}_{0}(\mathrm{t})+\mu_{1} \mathrm{P}_{2}(\mathrm{t})+\xi(1-\mathrm{p}) \sum_{\mathrm{r}=1}^{\mathrm{N}-1} \mathrm{c}_{\mathrm{r}} \mathrm{P}_{(1+\mathrm{r})}(\mathrm{t}) \\
& \mathrm{P}_{2}^{\prime}(\mathrm{t})=-\left(\lambda+\mu_{1}+\mu_{2}+\xi\right) \mathrm{P}_{2}(\mathrm{t})+\lambda \mathrm{P}_{1,0}(\mathrm{t})+\lambda \mathrm{P}_{0,1}(\mathrm{t})+\left(\mu_{1}+\mu_{2}\right) \mathrm{P}_{3}(\mathrm{t})+\xi \sum_{\mathrm{r}=1}^{\mathrm{N}-2} \mathrm{c}_{\mathrm{r}} \mathrm{P}_{(2+\mathrm{r})}(\mathrm{t}) \\
& \mathrm{P}_{\mathrm{n}}^{\prime}(\mathrm{t})=-\left(\lambda+\mu_{1}+\mu_{2}+\xi\right) \mathrm{P}_{\mathrm{n}}(\mathrm{t})+\lambda \mathrm{P}_{(\mathrm{n}-1)}(\mathrm{t})+\left(\mu_{1}+\mu_{2}\right) \mathrm{P}_{(\mathrm{n}+1)}(\mathrm{t})+\xi \sum_{\mathrm{r}=1}^{\mathrm{N}-\mathrm{n}} \mathrm{c}_{\mathrm{r}} \mathrm{P}_{(\mathrm{n}+\mathrm{r})}(\mathrm{t}), \mathrm{n}=3,4,5, \ldots, N-1
\end{aligned}
$$

$\mathrm{P}_{\mathrm{N}}^{\prime}(\mathrm{t})=-\left(\mu_{1}+\mu_{2}+\xi\right) \mathrm{P}_{\mathrm{N}}(\mathrm{t})+\lambda \mathrm{P}_{(\mathrm{N}-1)}(\mathrm{t})$

Taking, Laplace Transform of equations (1) to (6) w.r.t. ' $t$ ', we have

$$
\begin{aligned}
& \mathrm{sP}_{0}^{*}(\mathrm{~s})=1-\lambda \mathrm{P}_{0}^{*}(\mathrm{~s})+\mu_{1} \mathrm{P}_{1,0}^{*}(\mathrm{~s})+\mu_{2} \mathrm{P}_{0,1}^{*}(\mathrm{~s})+\xi \sum_{\mathrm{n}=1}^{\mathrm{N}} \sum_{\mathrm{r}=\mathrm{n}}^{\mathrm{N}} \mathrm{c}_{\mathrm{r}} \mathrm{P}_{\mathrm{n}}^{*}(\mathrm{~s}) \\
& \mathrm{sP}_{1,0}^{*}(\mathrm{~s})=-\left(\lambda+\mu_{1}+\xi\right) \mathrm{P}_{1,0}^{*}(\mathrm{~s})+\lambda \mathrm{p} \mathrm{P}_{0}^{*}(\mathrm{~s})+\mu_{2} \mathrm{P}_{2}^{*}(\mathrm{~s})+\xi \mathrm{p} \sum_{\mathrm{r}=1}^{\mathrm{N}-1} \mathrm{c}_{\mathrm{r}} \mathrm{P}_{[1+\mathrm{r}]}^{*}(\mathrm{~s}) \\
& \mathrm{sP}_{0,1}^{*}(\mathrm{~s})=-\left(\lambda+\mu_{2}+\xi\right) \mathrm{P}_{0,1}^{*}(\mathrm{~s})+\lambda(1-\mathrm{p}) \mathrm{P}_{0}^{*}(\mathrm{~s})+\mu_{1} \mathrm{P}_{2}^{*}(\mathrm{~s})+\xi(1-\mathrm{p}) \sum_{\mathrm{r}=1}^{\mathrm{N}-1} \mathrm{c}_{\mathrm{r}} \mathrm{P}_{[1+\mathrm{r}]}^{*}(\mathrm{~s}) \\
& \mathrm{sP}_{2}^{*}(\mathrm{~s})=-\left(\lambda+\mu_{1}+\mu_{2}+\xi\right) \mathrm{P}_{2}^{*}(\mathrm{~s})+\lambda \mathrm{P}_{1,0}^{*}(\mathrm{~s})+\lambda \mathrm{P}_{0,1}^{*}(\mathrm{~s})+\left(\mu_{1}+\mu_{2}\right) \mathrm{P}_{3}^{*}(\mathrm{~s})+\xi \sum_{\mathrm{r}=1}^{\mathrm{N}-2} \mathrm{c}_{\mathrm{r}} \mathrm{P}_{[2+\mathrm{r}]}^{*}(\mathrm{~s})(10) \\
& \mathrm{sP}_{\mathrm{n}}^{*}(\mathrm{~s})=-\left(\lambda+\mu_{1}+\mu_{2}+\xi\right) \mathrm{P}_{\mathrm{n}}^{*}(\mathrm{~s})+\lambda \mathrm{P}_{(\mathrm{n}-1)}^{*}(\mathrm{~s})+\left(\mu_{1}+\mu_{2}\right) \mathrm{P}_{(\mathrm{n}+1)}^{*}(\mathrm{~s})+\xi \sum_{\mathrm{r}=1}^{\mathrm{N}-\mathrm{n}} \mathrm{c}_{\mathrm{r}} \mathrm{P}_{[\mathrm{n}+\mathrm{r}]}^{*}(\mathrm{~s}) \\
& \mathrm{sP}_{\mathrm{N}}^{*}(\mathrm{~s})=-\left(\mu_{1}+\mu_{2}+\xi\right) \mathrm{P}_{\mathrm{N}}^{*}(\mathrm{~s})+\lambda \mathrm{P}_{(\mathrm{N}-1)}^{*}(\mathrm{~s})
\end{aligned}
$$

Where

$$
\mathrm{P}_{\mathrm{n}}^{*}(\mathrm{~s})=\int_{0}^{\infty} \mathrm{e}^{-\mathrm{st}} \mathrm{P}_{\mathrm{n}}(\mathrm{t}) \mathrm{dt} \quad \text { and } \quad \mathbf{P}_{\mathrm{O}}(\mathrm{O})=1
$$

Solving equations (11) and (12) recursively, we have

$$
\begin{aligned}
& P_{n}^{*}(s)=\rho^{-N}\left\{\rho^{n}+\sum_{i=1}^{\left[\frac{-3+\sqrt{9+8(N-n)}}{2}\right]}\left[\frac{2(N-n)-(i(i-1))}{4}\right] \sum_{1_{0}=i}^{i-1}\left(\sum_{j=1}^{1_{j-1}-1} \sum_{1_{j}=(i-j)}^{i-1} \prod_{m=0}^{\left[A_{m}\right]}\left(\sum_{k_{(m+1)}=k_{m}+1}^{\left.A_{m}\right]} \eta \rho^{L_{i}+n} D_{i}\right\} P_{N}^{*}(s)\right.\right. \\
& \mathrm{n}=3,4, \ldots \ldots, \mathrm{N}-1
\end{aligned}
$$

Where 


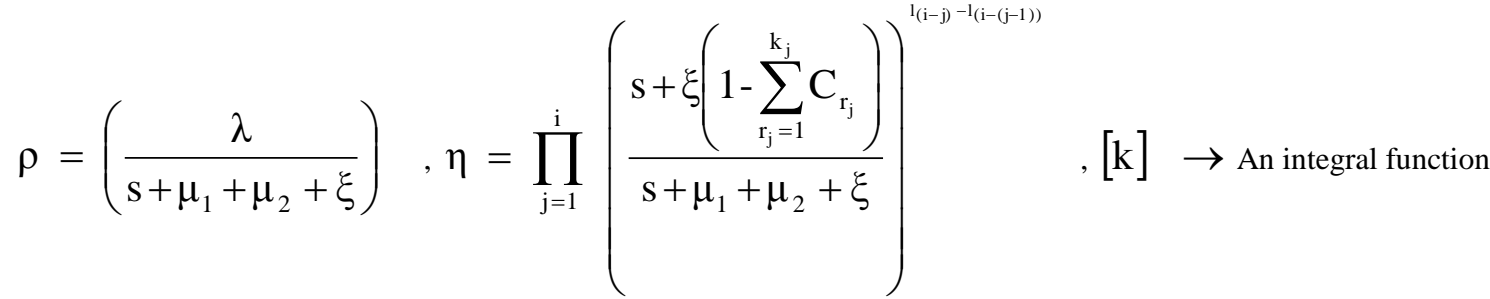

$$
\begin{aligned}
& \prod_{j}^{i}=1 \text { and } \sum_{j}^{i}=0 \text { for } \mathrm{i}<\mathrm{j}, \mathrm{k}_{0}=0 \text {, } \\
& A_{m}=\frac{N-n-(i-m) 1_{0}+\sum_{a=1}^{i-m-1} 1_{a}-k_{m} 1_{(i-m)}-\sum_{b=1}^{m-1}\left(k_{(m-b)}-k_{(m-(b-1))}\right) 1_{(i+b-m)}}{1_{0}-1_{(i-m)}} \\
& \mathrm{L}_{\mathrm{i}}=\sum_{\mathrm{j}=1}^{\mathrm{i}}\left(\mathrm{l}_{(\mathrm{i}-\mathrm{j})}-\mathrm{l}_{(\mathrm{i}-\mathrm{j}-1))}\right) \mathrm{k}_{\mathrm{j}}, \mathrm{l}_{\mathrm{j}}=\left\{\begin{array}{ll}
0 & \text { if } \mathrm{j}=\mathrm{i} \\
1_{\mathrm{j}} & \text { if } 1 \leq \mathrm{j}<\mathrm{i}
\end{array} \text { and } \mathrm{D}_{\mathrm{i}}=\prod_{\mathrm{j}=1}^{\mathrm{i}}\left(\begin{array}{c}
\mathrm{N}-\mathrm{n}-\mathrm{L}_{\mathrm{i}}-\mathrm{l}_{(\mathrm{i}-(\mathrm{j}-1))} \\
\mathrm{l}_{(\mathrm{i}-\mathrm{j})}-\mathrm{l}_{(\mathrm{i}-(\mathrm{j}-1))}
\end{array}\right)\right.
\end{aligned}
$$

Now, the probabilities $\mathrm{P}_{0}^{*}(\mathrm{~s}), \mathrm{P}_{0,1}^{*}(\mathrm{~s}), \mathrm{P}_{1,0}^{*}(\mathrm{~s})$, and $\mathrm{P}_{2}^{*}(\mathrm{~s})$ remain to be found. For this, we consider the equations (7) - (10). After simplification, we have

$\mathrm{P}_{0}^{*}(\mathrm{~s})=\frac{\left(\mathrm{B}_{1} \rho^{-\mathrm{N}} \mathrm{P}_{\mathrm{N}}^{*}(\mathrm{~s})\right)+\left(\frac{(\mathrm{s}+\xi)}{\mathrm{s}}\left(\lambda \mathrm{Q}_{1}-\mathrm{RR}_{1} \mathrm{R}_{2}\right)\right)}{\mathrm{G}}$

$$
\mathrm{P}_{1,0}^{*}(\mathrm{~s})=\frac{\left(\mathrm{B}_{2} \rho^{-\mathrm{N}} \mathrm{P}_{\mathrm{N}}^{*}(\mathrm{~s})\right)+\frac{\lambda(\mathrm{s}+\xi)}{\mathrm{s}}\left(\lambda \mathrm{U}_{1}-\mathrm{pRR} \mathrm{R}_{2}\right)}{\mathrm{G}}
$$

$$
\mathrm{P}_{0,1}^{*}(\mathrm{~s})=\frac{\left(\mathrm{B}_{3} \rho^{-\mathrm{N}} \mathrm{P}_{\mathrm{N}}^{*}(\mathrm{~s})\right)-\frac{\lambda(\mathrm{s}+\xi)}{\mathrm{s}}\left(\lambda \mathrm{U}_{1}+(1-\mathrm{p}) \mathrm{RR}_{1}\right)}{\mathrm{G}}
$$

$$
\mathrm{P}_{2}^{*}(\mathrm{~s})=\frac{\left(\mathrm{B}_{4} \rho^{-\mathrm{N}} \mathrm{P}_{\mathrm{N}}^{*}(\mathrm{~s})\right)-\left(\frac{\lambda^{2}(\mathrm{~s}+\xi) \mathrm{T}_{1}}{\mathrm{~s}}\right)}{\mathrm{G}}
$$

In terms of unknown $\mathrm{P}_{\mathrm{N}}^{*}(\mathrm{~s})$ and the constants:

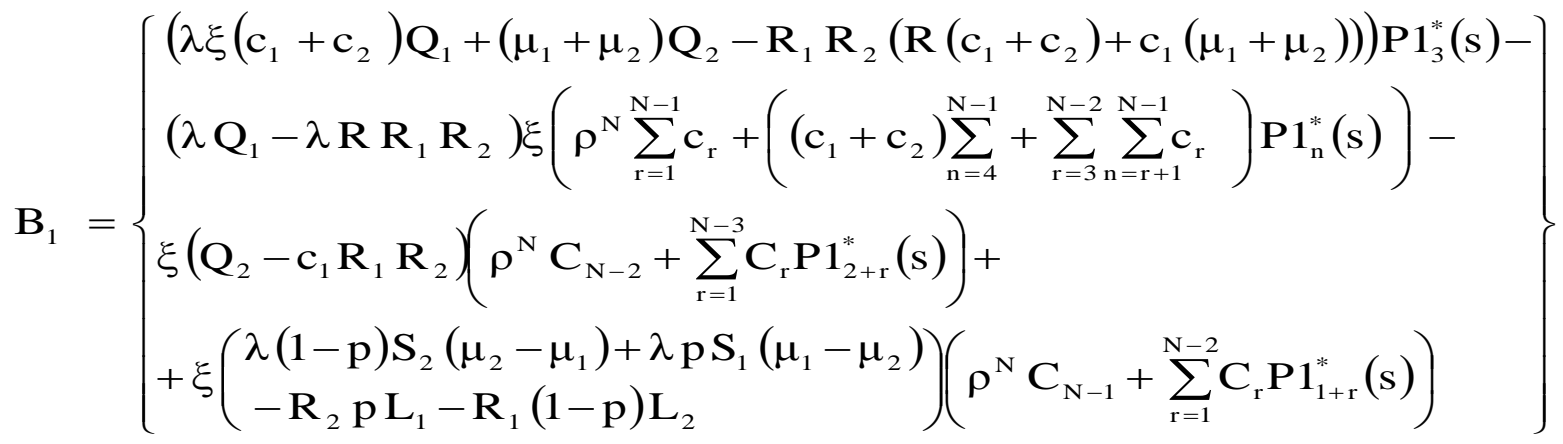




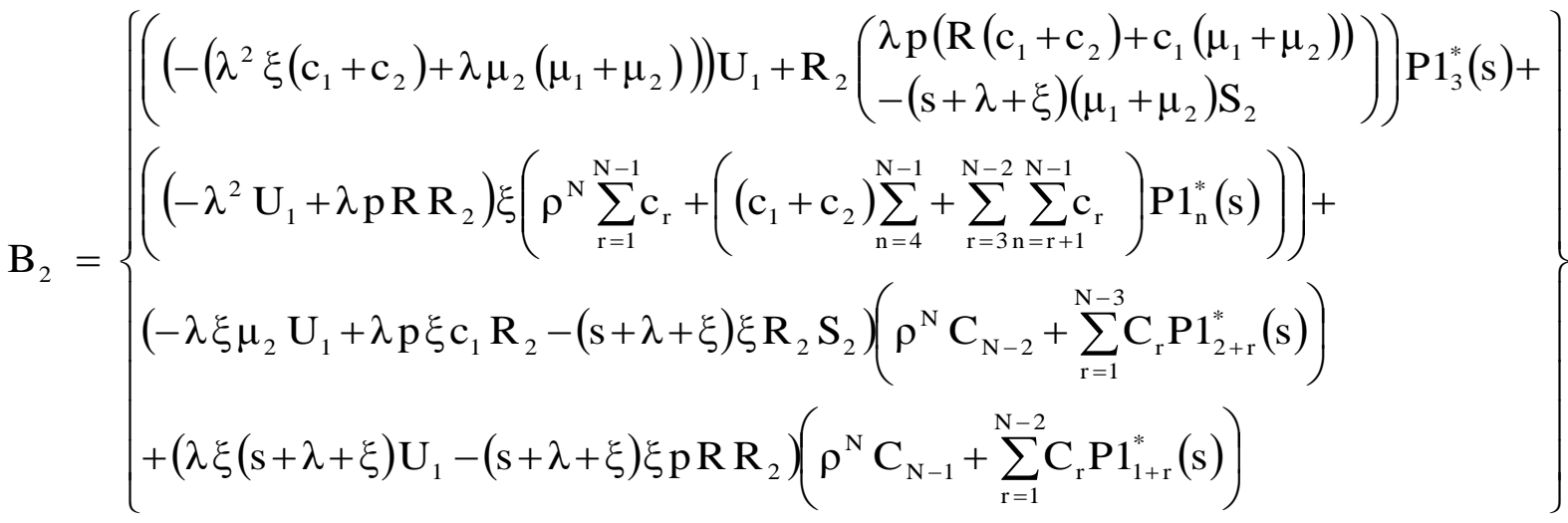

$$
\begin{aligned}
& \left(\left(\left(\left(\lambda^{2} \xi\left(\mathrm{c}_{1}+\mathrm{c}_{2}\right)+\lambda \mu_{1}\left(\mu_{1}+\mu_{2}\right)\right)\right) \mathrm{U}_{1}+\mathrm{R}_{1}\left(\begin{array}{l}
\lambda(1-\mathrm{p})\left(\mathrm{R}\left(\mathrm{c}_{1}+\mathrm{c}_{2}\right)+\mathrm{c}_{1}\left(\mu_{1}+\mu_{2}\right)\right) \\
-(\mathrm{s}+\lambda+\xi)\left(\mu_{1}+\mu_{2}\right) \mathrm{S}_{1}
\end{array}\right)\right) \mathrm{P}_{3}^{*}(\mathrm{~s})+\right. \\
& \mathrm{B}_{3}=\left\{\begin{array}{l}
\left(\left(\lambda^{2} \mathrm{U}_{1}+\lambda(1-\mathrm{p}) \mathrm{R} \mathrm{R}_{1}\right) \xi\left(\rho^{\mathrm{N}} \sum_{\mathrm{r}=1}^{\mathrm{N}-1} \mathrm{c}_{\mathrm{r}}+\left(\left(\mathrm{c}_{1}+\mathrm{c}_{2}\right) \sum_{\mathrm{n}=4}^{\mathrm{N}-1}+\sum_{\mathrm{r}=3 \mathrm{n}=\mathrm{r}+1}^{\mathrm{N}-2} \sum_{\mathrm{r}}^{\mathrm{N}-1} \mathrm{c}_{\mathrm{r}}\right) \mathrm{P} 1_{\mathrm{n}}^{*}(\mathrm{~s})\right)\right)+ \\
\left(\lambda \xi \mu_{1} \mathrm{U}_{1}+\lambda(1-\mathrm{p}) \xi \mathrm{c}_{1} \mathrm{R}_{1}-(\mathrm{s}+\lambda+\xi) \xi \mathrm{R}_{1} \mathrm{~S}_{1}\right)\left(\rho^{\mathrm{N}} \mathrm{C}_{\mathrm{N}-2}+\sum_{\mathrm{r}=1}^{\mathrm{N}-3} \mathrm{C}_{\mathrm{r}} \mathrm{P} 1_{2+\mathrm{r}}^{*}(\mathrm{~s})\right)
\end{array}\right. \\
& +\left(-\lambda \xi(\mathrm{s}+\lambda+\xi) \mathrm{U}_{1}-(\mathrm{s}+\lambda+\xi) \xi(1-\mathrm{p}) \mathrm{R}_{1}\right)\left(\rho^{\mathrm{N}} \mathrm{C}_{\mathrm{N}-1}+\sum_{\mathrm{r}=1}^{\mathrm{N}-2} \mathrm{C}_{\mathrm{r}} \mathrm{P}_{1+\mathrm{r}}^{*}(\mathrm{~s})\right) \\
& \mathrm{B}_{4}=\left\{\begin{array}{l}
\left(\lambda^{2} \xi\left(\mathrm{c}_{1}+\mathrm{c}_{2}\right) \mathrm{T}_{1}+\lambda\left(\mu_{1}+\mu_{2}\right) \mathrm{T}_{2}-(\mathrm{s}+\lambda+\xi)\left(\mu_{1}+\mu_{2}\right) \mathrm{R}_{1} \mathrm{R}_{2}\right) \mathrm{P} 1_{3}^{*}(\mathrm{~s}) \\
+\lambda^{2} \mathrm{~T}_{1} \xi\left(\rho^{\mathrm{N}} \sum_{\mathrm{r}=1}^{\mathrm{N}-1} \mathrm{c}_{\mathrm{r}}+\left(\left(\mathrm{c}_{1}+\mathrm{c}_{2}\right) \sum_{\mathrm{n}=4}^{\mathrm{N}-1}+\sum_{\mathrm{r}=3}^{\mathrm{N}-2} \sum_{\mathrm{n}=\mathrm{r}+1}^{\mathrm{N}-1} \mathrm{c}_{\mathrm{r}}\right) \mathrm{P} 1_{\mathrm{n}}^{*}(\mathrm{~s})\right)+ \\
\xi\left(\lambda \mathrm{T}_{2}-(\mathrm{s}+\lambda+\xi) \mathrm{R}_{1} \mathrm{R}_{2}\right)\left(\rho^{\mathrm{N}} \mathrm{C}_{\mathrm{N}-2}+\sum_{\mathrm{r}=1}^{\mathrm{N}-3} \mathrm{C}_{\mathrm{r}} \mathrm{P} 1_{2+\mathrm{r}}^{*}(\mathrm{~s})\right) \\
-\lambda \xi(\mathrm{s}+\lambda+\xi) \mathrm{T}_{1}\left(\rho^{\mathrm{N}} \mathrm{C}_{\mathrm{N}-1}+\sum_{\mathrm{r}=1}^{\mathrm{N}-2} \mathrm{C}_{\mathrm{r}} \mathrm{P} 1_{1+\mathrm{r}}^{*}(\mathrm{~s})\right)
\end{array}\right\}
\end{aligned}
$$$$
\mathrm{G}=\left\{\begin{array}{l}
\lambda \mathrm{S}_{1}\left(\lambda \mathrm{p}\left(\mu_{2}-\mu_{1}\right)+(\mathrm{s}+\lambda+\xi) \mathrm{R}_{1}\right)+\lambda \mathrm{S}_{2}\left(\lambda(1-\mathrm{p})\left(\mu_{1}-\mu_{2}\right)+(\mathrm{s}+\lambda+\xi) \mathrm{R}_{2}\right)+\lambda \mathrm{pL} \mathrm{L}_{1} \\
+\lambda(1-\mathrm{p}) \mathrm{L}_{2}-(\mathrm{s}+\lambda+\xi) \mathrm{R}_{1} \mathrm{R}_{2}
\end{array}\right\}
$$

And

$$
\begin{aligned}
& \mathrm{R}=\left(\mathrm{s}+\lambda+\mu_{1}+\mu_{2}+\xi\right), \mathrm{R}_{1}=\left(\mathrm{s}+\lambda+\mu_{1}+\xi\right), \mathrm{R}_{2}=\left(\mathrm{s}+\lambda+\mu_{2}+\xi\right), \mathrm{S}_{1}=\left(\mu_{1}+\xi(1-\mathrm{p}) \mathrm{c}_{1}\right), \\
& \mathrm{S}_{2}=\left(\mu_{2}+\xi \mathrm{pc}_{1}\right), \mathrm{L}_{1}=\left(\mathrm{R} \mu_{1}-\lambda \xi \mathrm{c}_{1}\right), \mathrm{L}_{2}=\left(\mathrm{R} \mu_{2}-\lambda \xi \mathrm{c}_{1}\right), \mathrm{T}_{1}=\left(\mathrm{p} \mathrm{R}_{2}+(1-\mathrm{p}) \mathrm{R}_{1}\right), \\
& \mathrm{T}_{2}=\left(\mu_{1} \mathrm{pR}_{2}+\mu_{2}(1-\mathrm{p}) \mathrm{R}_{1}\right), \mathrm{Q}_{1}=\left(\mathrm{S}_{2} \mathrm{R}_{2}+\mathrm{S}_{1} \mathrm{R}_{1}\right), \mathrm{Q}_{2}=\left(\mu_{1} \mathrm{~S}_{2} \mathrm{R}_{2}+\mu_{2} \mathrm{~S}_{1} \mathrm{R}_{1}\right), \\
& \mathrm{U}_{1}=\left(\mathrm{pS} \mathrm{S}_{1}-(1-\mathrm{p}) \mathrm{S}_{2}\right)
\end{aligned}
$$

Where

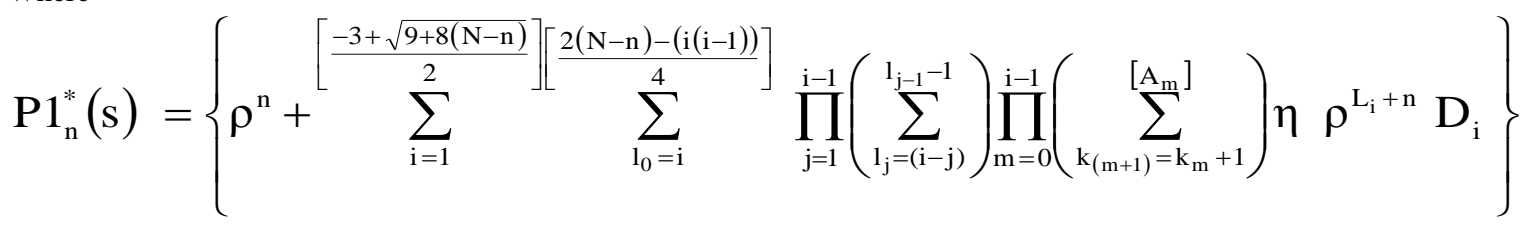


Using normalization condition, we have

$$
P_{N}^{*}(s)=\frac{\left(G-(s+\xi)\left(\lambda Q_{1}-R_{1} R_{2}-(R+\lambda) \lambda T_{1}\right)\right)}{s \rho^{-N}\left\{+G \sum_{n=3}^{N}\left\{\rho^{n}+\sum_{i=1}^{\left(B_{1}+B_{2}+B_{3}+B_{4}\right)}\right]\left[\frac{-3+\sqrt{9+8(N-n)}}{2}\left[\frac{2(N-n)-(i(i-1))}{4}\right] \prod_{l_{0}=i}^{4} \prod_{j=1}^{i-1}\left(\sum_{1_{j}=(i-j)}^{1_{j-1}-1}\right) \prod_{m=0}^{i-1}\left(\sum_{k(m+1)=k_{m}+1}^{\left[A_{m}\right]} \eta \rho^{L_{i}+n} D_{i}\right\}\right.\right.}
$$

After using (18), equations (14)-(17) becomes:

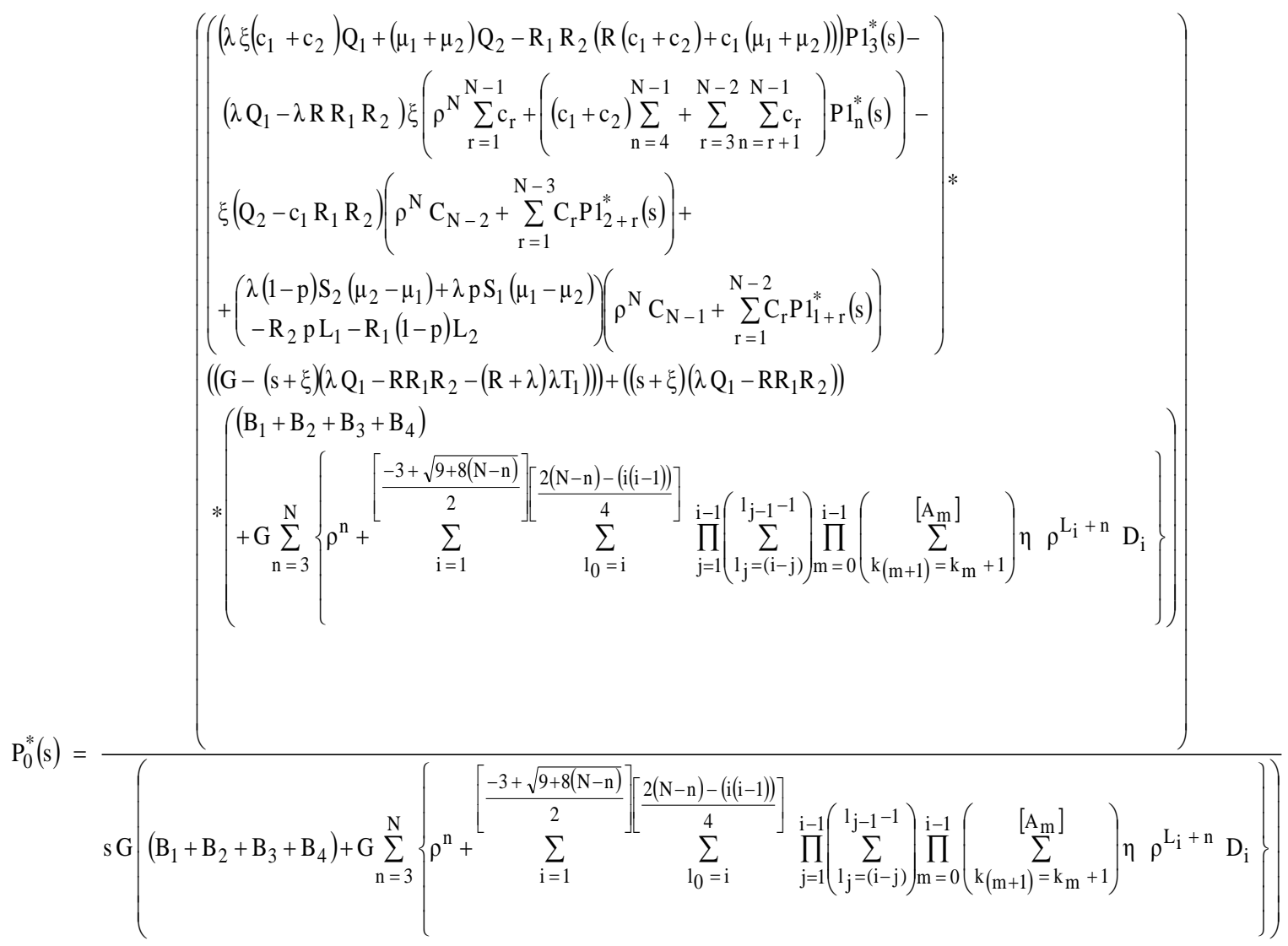




$$
\begin{aligned}
& \left.\int\left(\left(-\left(\lambda^{2} \xi\left(\mathrm{c}_{1}+\mathrm{c}_{2}\right)+\lambda \mu_{2}\left(\mu_{1}+\mu_{2}\right)\right)\right) \mathrm{U}_{1}+\mathrm{R}_{2}\left(\begin{array}{l}
\lambda \mathrm{p}\left(\mathrm{R}\left(\mathrm{c}_{1}+\mathrm{c}_{2}\right)+\mathrm{c}_{1}\left(\mu_{1}+\mu_{2}\right)\right) \\
-(\mathrm{s}+\lambda+\xi)\left(\mu_{1}+\mu_{2}\right) \mathrm{S}_{2}
\end{array}\right)\right) \mathrm{P}_{3}^{*}(\mathrm{~s})+\right) \\
& \left\{\left(\left(-\lambda^{2} \mathrm{U}_{1}+\lambda \mathrm{pRR} \mathrm{R}_{2}\right) \xi\left(\rho^{\mathrm{N}} \sum_{\mathrm{r}=1}^{\mathrm{N}-1} \mathrm{c}_{\mathrm{r}}+\left(\left(\mathrm{c}_{1}+\mathrm{c}_{2}\right) \sum_{\mathrm{n}=4}^{\mathrm{N}-1}+\sum_{\mathrm{r}=3 \mathrm{n}=\mathrm{r}+1}^{\mathrm{N}-2} \sum_{\mathrm{r}}^{\mathrm{N}-1} \mathrm{c}_{\mathrm{r}}\right) \mathrm{Pl}_{\mathrm{n}}^{*}(\mathrm{~s})\right)\right)+\right. \\
& \left(-\lambda \xi \mu_{2} \mathrm{U}_{1}+\lambda \mathrm{p} \xi \mathrm{c}_{1} \mathrm{R}_{2}-(\mathrm{s}+\lambda+\xi) \xi \mathrm{R}_{2} \mathrm{~S}_{2}\right)\left(\rho^{\mathrm{N}} \mathrm{C}_{\mathrm{N}-2}+\sum_{\mathrm{r}=1}^{\mathrm{N}-3} \mathrm{C}_{\mathrm{r}} \mathrm{P}_{2+\mathrm{r}}^{*}(\mathrm{~s})\right) \\
& +\left(\lambda \xi(\mathrm{s}+\lambda+\xi) \mathrm{U}_{1}-(\mathrm{s}+\lambda+\xi) \xi \mathrm{pRR} \mathrm{R}_{2}\right)\left(\rho^{\mathrm{N}} \mathrm{C}_{\mathrm{N}-1}+\sum_{\mathrm{r}=1}^{\mathrm{N}-2} \mathrm{C}_{\mathrm{r}} \mathrm{P}_{1+\mathrm{r}}^{*}(\mathrm{~s})\right) \\
& \left(\left(\mathrm{G}-(\mathrm{s}+\xi)\left(\lambda \mathrm{Q}_{1}-\mathrm{RR}_{1} \mathrm{R}_{2}-(\mathrm{R}+\lambda) \lambda \mathrm{T}_{1}\right)\right)\right)+
\end{aligned}
$$

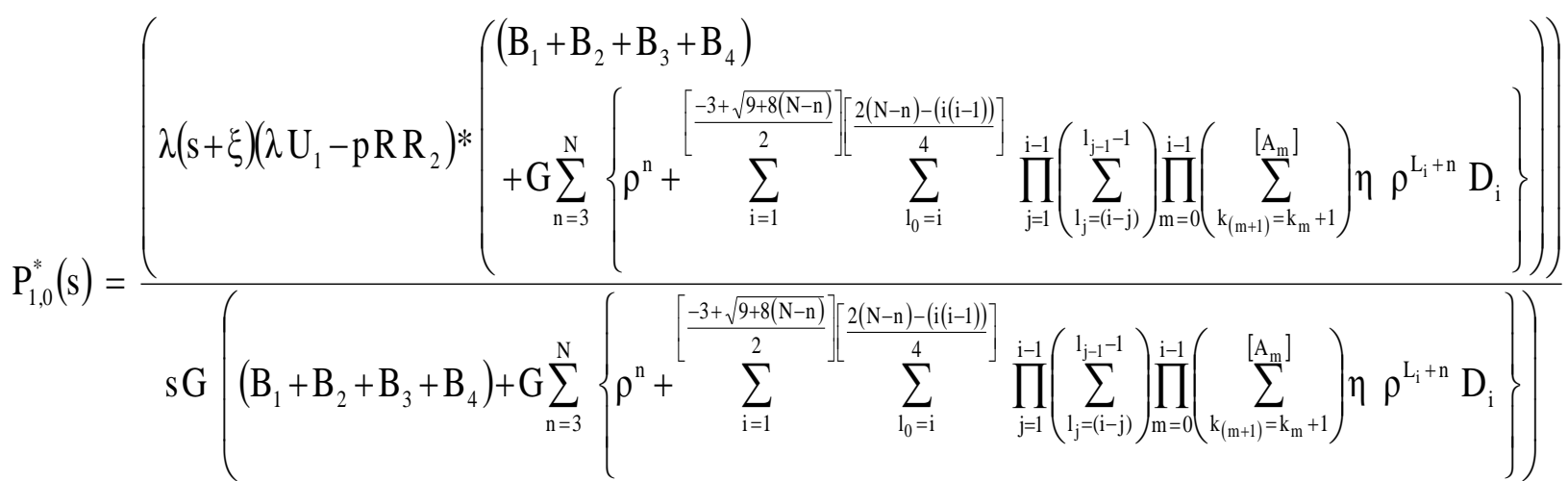

$$
\begin{aligned}
& \left\{\begin{array}{l}
\left(\left(\left(\lambda^{2} \xi\left(\mathrm{c}_{1}+\mathrm{c}_{2}\right)+\lambda \mu_{1}\left(\mu_{1}+\mu_{2}\right)\right)\right) \mathrm{U}_{1}+\mathrm{R}_{1}\left(\begin{array}{l}
\lambda(1-\mathrm{p})\left(\mathrm{R}\left(\mathrm{c}_{1}+\mathrm{c}_{2}\right)+\mathrm{c}_{1}\left(\mu_{1}+\mu_{2}\right)\right) \\
-(\mathrm{s}+\lambda+\xi)\left(\mu_{1}+\mu_{2}\right) \mathrm{S}_{1}
\end{array}\right)\right) \mathrm{Pl}_{3}^{*}(\mathrm{~s})+ \\
\left(\left(\lambda^{2} \mathrm{U}_{1}+\lambda(1-\mathrm{p}) \mathrm{R} \mathrm{R}_{1}\right) \xi\left(\rho^{\mathrm{N}} \sum_{\mathrm{r}=1}^{\mathrm{N}-1} \mathrm{c}_{\mathrm{r}}+\left(\left(\mathrm{c}_{1}+\mathrm{c}_{2}\right) \sum_{\mathrm{n}=4}^{\mathrm{N}-1}+\sum_{\mathrm{r}=3 \mathrm{n}=\mathrm{r}+1}^{\mathrm{N}-2} \sum_{\mathrm{r}}^{\mathrm{N}-1} \mathrm{c}_{\mathrm{r}}\right) \mathrm{Pl}_{\mathrm{n}}^{*}(\mathrm{~s})\right)\right)+ \\
\left(\lambda \xi \mu_{1} \mathrm{U}_{1}+\lambda(1-\mathrm{p}) \xi \mathrm{c}_{1} \mathrm{R}_{1}-(\mathrm{s}+\lambda+\xi) \xi \mathrm{R}_{1} \mathrm{~S}_{1}\right)\left(\rho^{\mathrm{N}} \mathrm{C}_{\mathrm{N}-2}+\sum_{\mathrm{r}=1}^{\mathrm{N}-3} \mathrm{C}_{\mathrm{r}} \mathrm{Pl}_{2+\mathrm{r}}^{*}(\mathrm{~s})\right) \\
+\left(-\lambda \xi(\mathrm{s}+\lambda+\xi) \mathrm{U}_{1}-(\mathrm{s}+\lambda+\xi) \xi(1-\mathrm{p}) \mathrm{RR}_{1}\right)\left(\rho^{\mathrm{N}} \mathrm{C}_{\mathrm{N}-1}+\sum_{\mathrm{r}=1}^{\mathrm{N}-2} \mathrm{C}_{\mathrm{r}} \mathrm{Pl}_{1+\mathrm{r}}^{*}(\mathrm{~s})\right)
\end{array}\right\} * \\
& \left(\left(\mathrm{G}-(\mathrm{s}+\xi)\left(\lambda \mathrm{Q}_{1}-\mathrm{RR}_{1} \mathrm{R}_{2}-(\mathrm{R}+\lambda) \lambda \mathrm{T}_{1}\right)\right)\right)-
\end{aligned}
$$

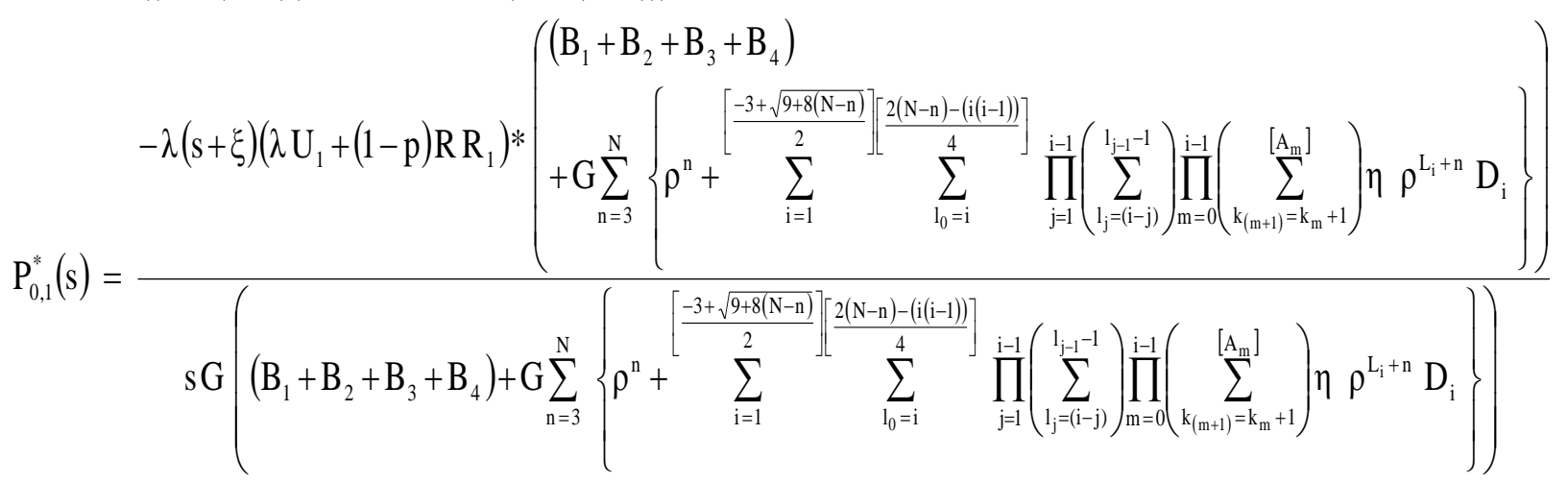




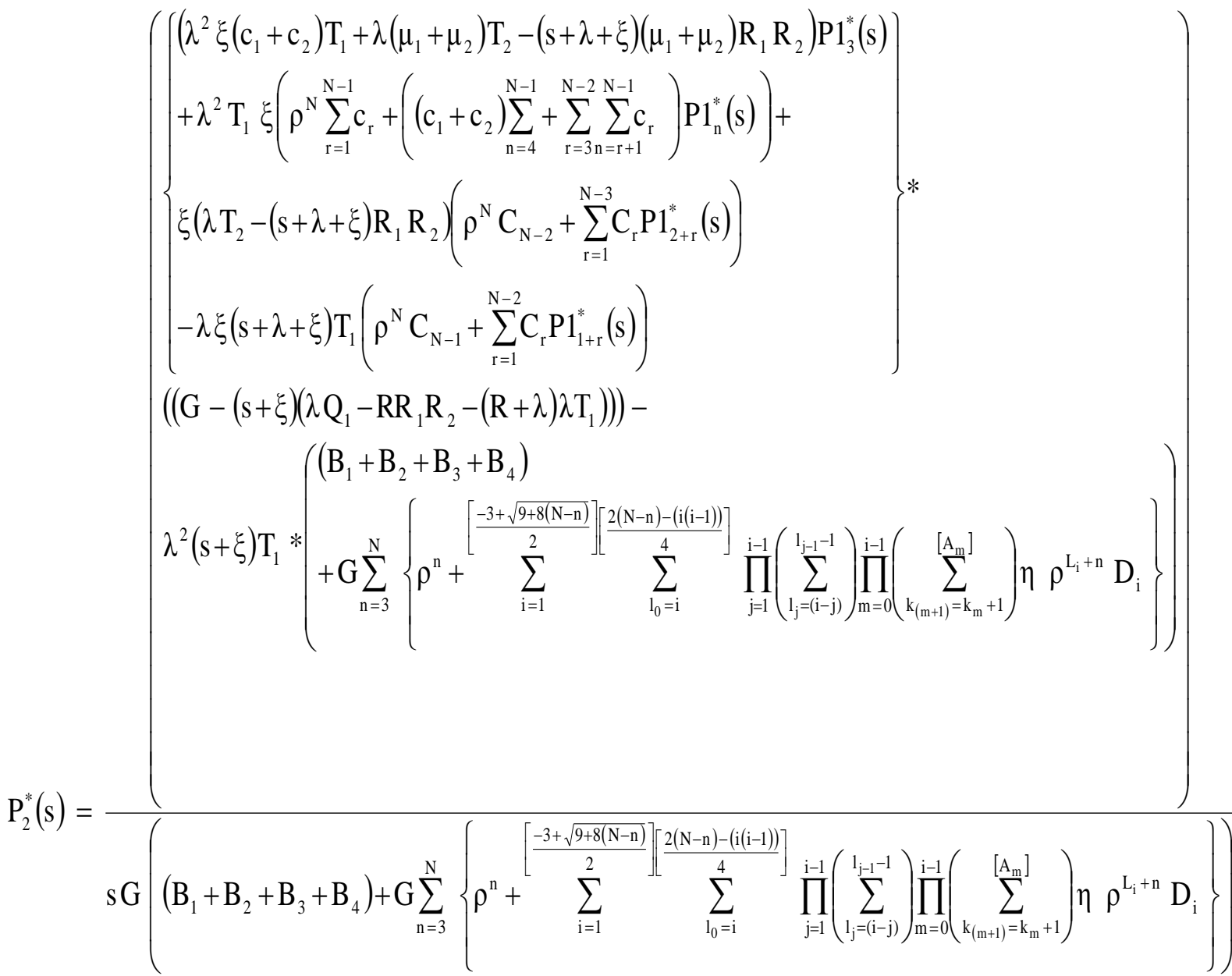

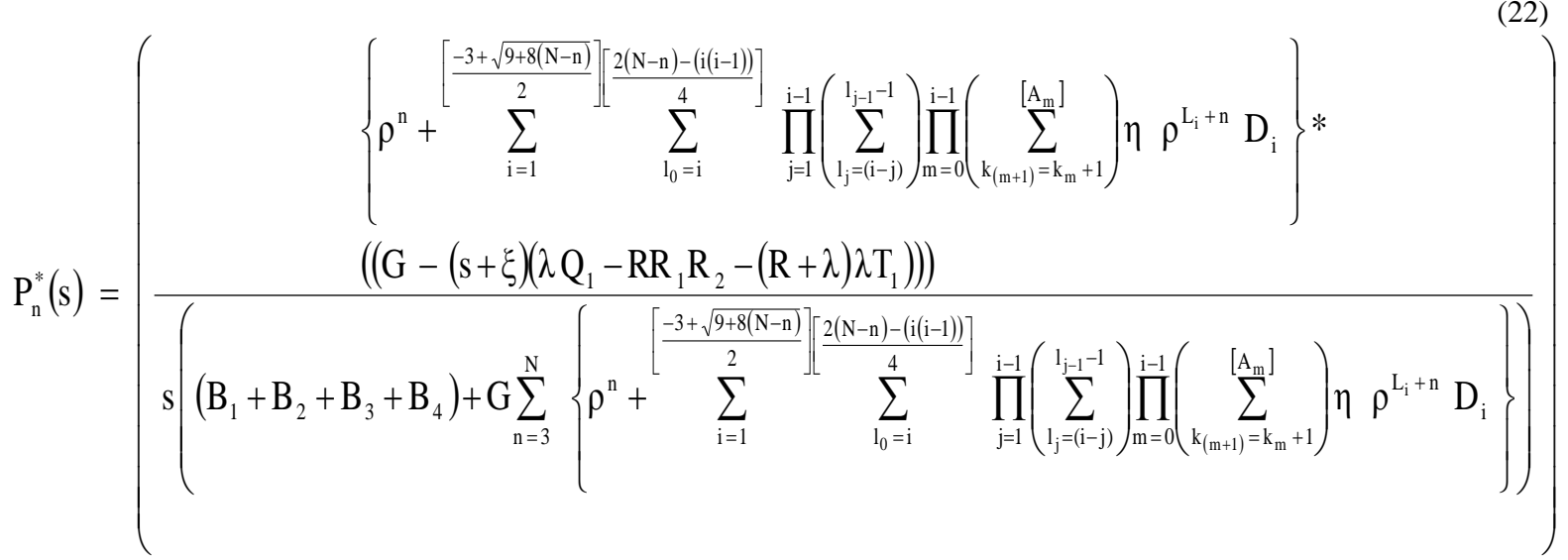

After taking Laplace inverse of (19) to (23), we can find all the probabilities.

\section{SIMULATION RESULTS}

We obtain numerically the various measures of performance of this model by using simulation technique. The simulation analysis of the queueing model under investigation is carried out by using a computer program written in $\mathrm{C}$ language. The simulation results have been shown in tables 1-6. In tables 13 , the simulation results are obtained by assuming that the catastrophic intensity follows the uniform distribution while in tables 4-6, the simulation results are obtained by assuming that the catastrophic intensity follows the modified binomial distribution. 
Table-1

Simulation results of an $M / M / 2 / N$ queueing model with two heterogeneous servers subject to uniformly distributed catastrophic intensity Effect of change in mean inter arrival time $(1 / \lambda)$ Mean service time of server $1=6$

minutes, Mean service time of server $2=8$ minutes, mean inter catastrophe time $=100$ minutes, simulation length $=$ 480, $N=5$

\begin{tabular}{|l|l|l|l|l|}
\hline $\begin{array}{l}\text { Mean } \\
\text { inter } \\
\text { arrival } \\
\text { time }\end{array}$ & $\begin{array}{l}\text { Averag } \\
\text { e delay } \\
\text { in } \\
\text { queue }\end{array}$ & $\begin{array}{l}\text { Avera } \\
\text { ge No. } \\
\text { in } \\
\text { queue }\end{array}$ & $\begin{array}{l}\text { Server 1 } \\
\text { Utilization }\end{array}$ & $\begin{array}{l}\text { Server 2 } \\
\text { Utilization }\end{array}$ \\
\hline 1 & 12.1858 & 3.9533 & 0.9154 & 0.9154 \\
\hline 2 & 8.8038 & 0.1333 & 0.0643 & 0.0669 \\
\hline 3 & 6.6864 & 0.7411 & 0.3213 & 0.3307 \\
\hline 4 & 8.7736 & 0.5198 & 0.2050 & 0.2056 \\
\hline 5 & 4.0348 & 0.3452 & 0.2650 & 0.2693 \\
\hline 6 & 3.1294 & 0.0000 & 0.0051 & 0.0089 \\
\hline 7 & 2.3818 & 0.1015 & 0.1347 & 0.1396 \\
\hline 8 & 2.2160 & 0.0800 & 0.0720 & 0.0745 \\
\hline 9 & 2.2716 & 0.0629 & 0.2946 & 0.2335 \\
\hline 10 & 4.6215 & 0.0814 & 0.1076 & 0.1081 \\
\hline
\end{tabular}

Table-2

Simulation results of an $M / M / 2 / N$ queueing model with two heterogeneous servers subject to uniformly distributed catastrophic intensity Effect of change in mean service time of server $1\left(1 / \mu_{1}\right)$ Mean inter arrival time

= 2 minutes, Mean service time of server $2=5$ minutes, mean inter catastrophe time $=\mathbf{1 0 0}$ minutes, simulation length $=480, N=5$

\begin{tabular}{|c|l|l|l|l|}
\hline $\begin{array}{l}\text { Mean } \\
\text { service } \\
\text { time of } \\
\text { server 1 }\end{array}$ & $\begin{array}{l}\text { Averag } \\
\text { e delay } \\
\text { in } \\
\text { queue }\end{array}$ & $\begin{array}{l}\text { Average } \\
\text { No. in } \\
\text { queue }\end{array}$ & $\begin{array}{l}\text { Server 1 } \\
\text { Utilization }\end{array}$ & $\begin{array}{l}\text { Server 2 } \\
\text { Utilizati } \\
\text { on }\end{array}$ \\
\hline 1 & 0.5793 & 0.1374 & 0.2253 & 0.3426 \\
\hline 2 & 3.0729 & 0.6185 & 0.4241 & 0.4593 \\
\hline 3 & 4.7255 & 1.1797 & 0.5258 & 0.5459 \\
\hline 4 & 4.6241 & 0.1946 & 0.1213 & 0.1308 \\
\hline 5 & 7.3049 & 0.9741 & 0.3504 & 0.3509 \\
\hline 6 & 8.5041 & 1.5390 & 0.4739 & 0.4718 \\
\hline 7 & 8.8002 & 0.8844 & 0.2583 & 0.2566 \\
\hline 8 & 8.9714 & 1.0563 & 0.3111 & 0.3048 \\
\hline 9 & 11.7780 & 2.9511 & 0.7905 & 0.7789 \\
\hline 10 & 10.4079 & 1.7684 & 0.4886 & 0.4873 \\
\hline
\end{tabular}

Table-3

Simulation results of an $M / M / 2 / N$ queueing model with two heterogeneous servers subject to uniformly distributed catastrophic intensity Effect of change in mean service time of server $2\left(1 / \mu_{2}\right)$ Mean inter arrival time

= 2 minutes, Mean service time of server 1 = 5 minutes, mean inter catastrophe time $=100$ minutes, simulation length $=480, N=5$

\begin{tabular}{|c|c|c|c|c|}
\hline $\begin{array}{c}\text { Mean } \\
\text { service } \\
\text { time of } \\
\text { server 2 }\end{array}$ & $\begin{array}{c}\text { Average } \\
\text { delay in } \\
\text { queue }\end{array}$ & $\begin{array}{c}\text { Averag } \\
\text { e No. in } \\
\text { queue }\end{array}$ & $\begin{array}{c}\text { Server1 } \\
\text { Utilization }\end{array}$ & $\begin{array}{c}\text { Server 2 } \\
\text { Utilizati } \\
\text { on }\end{array}$ \\
\hline 1 & 0.6742 & 0.0479 & 0.0446 & 0.0321 \\
\hline 2 & 1.5226 & 0.4428 & 0.5231 & 0.4007 \\
\hline
\end{tabular}

\begin{tabular}{|c|c|c|c|c|}
\hline 3 & 4.1287 & 0.2043 & 0.1320 & 0.1174 \\
\hline 4 & 5.1984 & 0.5185 & 0.2159 & 0.2143 \\
\hline 5 & 7.3049 & 0.9741 & 0.3504 & 0.3509 \\
\hline 6 & 7.7834 & 0.7722 & 0.2613 & 0.2628 \\
\hline 7 & 8.1628 & 1.0034 & 0.3231 & 0.3290 \\
\hline 8 & 11.6083 & 2.9928 & 0.7859 & 0.8010 \\
\hline 9 & 10.2921 & 2.6730 & 0.7819 & 0.7713 \\
\hline 10 & 8.9250 & 0.6030 & 0.1918 & 0.1989 \\
\hline
\end{tabular}

Table-4

Simulation results of an $\mathrm{M} / \mathrm{M} / 2 / \mathrm{N}$ queueing model with two heterogeneous servers subject to uniformly distributed catastrophic intensity Effect of change in mean inter catastrophic time $(1 / \xi)$ Mean inter arrival time =

2 minutes, Mean service time of server $1=6$ minutes, mean service time of server $2=8$ minutes, simulation length $=$ 480, $N=5$

\begin{tabular}{|l|l|l|l|l|}
\hline $\begin{array}{l}\text { Mean inter } \\
\text { catastrophi } \\
\text { c time }\end{array}$ & $\begin{array}{l}\text { Average } \\
\text { delay in } \\
\text { queue }\end{array}$ & $\begin{array}{l}\text { Averag } \\
\text { e No. in } \\
\text { queue }\end{array}$ & $\begin{array}{l}\text { Server 1 } \\
\text { Utilizatio } \\
\mathrm{n}\end{array}$ & $\begin{array}{l}\text { Server } \\
2 \\
\text { Utilizati } \\
\text { on }\end{array}$ \\
\hline 101 & 12.2138 & 3.0654 & 0.7954 & 0.7882 \\
\hline 102 & 9.7799 & 0.2531 & 0.1107 & 0.1097 \\
\hline 103 & 11.6128 & 2.1070 & 0.5458 & 0.5415 \\
\hline 104 & 9.3843 & 1.0284 & 0.3111 & 0.3113 \\
\hline 105 & 10.2497 & 1.3395 & 0.3909 & 0.3812 \\
\hline 106 & 9.9494 & 0.8560 & 0.2587 & 0.2556 \\
\hline 107 & 10.4430 & 1.0812 & 0.3006 & 0.2922 \\
\hline 108 & 9.8635 & 1.5633 & 0.4620 & 0.4479 \\
\hline 109 & 10.1851 & 1.0888 & 0.3306 & 0.3355 \\
\hline 110 & 12.1654 & 1.5348 & 0.4485 & 0.4424 \\
\hline
\end{tabular}

Table-5

Simulation results of an $M / M / 2 / N$ queueing model with two heterogeneous servers subject to modified binomially distributed catastrophic intensity.

Effect of change in mean inter arrival time $(1 / \lambda)$,

Mean service time of server 1 = 6 minutes, Mean service time of server $2=8$ minutes, mean inter catastrophe time $=$ 100 minutes, simulation length $=480, N=5$

\begin{tabular}{|l|l|l|l|l|}
\hline $\begin{array}{l}\text { Mean } \\
\text { inter } \\
\text { arrival } \\
\text { time }\end{array}$ & $\begin{array}{l}\text { Average } \\
\text { delay in } \\
\text { queue }\end{array}$ & $\begin{array}{l}\text { Average } \\
\text { No. in } \\
\text { queue }\end{array}$ & $\begin{array}{l}\text { Server 1 } \\
\text { Utilization }\end{array}$ & $\begin{array}{l}\text { Server 2 } \\
\text { Utilization }\end{array}$ \\
\hline 1 & 11.8935 & 4.2028 & 0.9806 & 0.9806 \\
\hline 2 & 9.0571 & 0.1052 & 0.0499 & 0.0505 \\
\hline 3 & 6.8118 & 0.8650 & 0.3777 & 0.3920 \\
\hline 4 & 8.7793 & 0.4342 & 0.1777 & 0.1792 \\
\hline 5 & 4.0348 & 0.3452 & 0.2630 & 0.2693 \\
\hline 6 & 3.1294 & 0.0000 & 0.0051 & 0.0089 \\
\hline 7 & 2.3051 & 0.1043 & 0.1419 & 0.1451 \\
\hline 8 & 2.2160 & 0.0800 & 0.0720 & 0.0745 \\
\hline 9 & 2.2716 & 0.0629 & 0.2946 & 0.2335 \\
\hline 10 & 4.4700 & 0.0922 & 0.0890 & 0.0896 \\
\hline
\end{tabular}


Table-6

Simulation results of an $M / M / 2 / N$ queueing model with two heterogeneous servers subject to modified binomially distributed catastrophic intensity.

Effect of change in mean service time of server $1\left(1 / \mu_{1}\right)$

Mean inter arrival time $=\mathbf{2}$ minutes, Mean service time

of server $2=5$ minutes, mean inter catastrophe time $=100$ minutes, simulation length $=\mathbf{4 8 0}, \mathrm{N}=5$

\begin{tabular}{|c|c|c|c|c|}
\hline $\begin{array}{c}\text { Mean } \\
\text { service } \\
\text { time of } \\
\text { server 1 }\end{array}$ & $\begin{array}{c}\text { Averag } \\
\text { e delay } \\
\text { in } \\
\text { queue }\end{array}$ & $\begin{array}{c}\text { Average } \\
\text { No. in } \\
\text { queue }\end{array}$ & $\begin{array}{c}\text { Server 1 1 } \\
\text { Utilizati } \\
\text { on }\end{array}$ & $\begin{array}{c}\text { Server 2 } \\
\text { Utilization }\end{array}$ \\
\hline 1 & 0.5793 & 0.1374 & 0.2253 & 0.3426 \\
\hline 2 & 3.1418 & 0.9103 & 0.4159 & 0.4500 \\
\hline 3 & 4.7255 & 1.1797 & 0.5258 & 0.5459 \\
\hline 4 & 4.4515 & 0.0916 & 0.0847 & 0.0970 \\
\hline 5 & 7.3563 & 0.8923 & 0.3362 & 0.3395 \\
\hline 6 & 8.9504 & 1.7259 & 0.5161 & 0.5192 \\
\hline 7 & 8.6378 & 0.7640 & 0.2275 & 0.2256 \\
\hline 8 & 8.9829 & 1.2061 & 0.3489 & 0.3417 \\
\hline 9 & 11.8682 & 3.4734 & 0.9425 & 0.9284 \\
\hline 10 & 10.8180 & 1.8999 & 0.5136 & 0.5116 \\
\hline
\end{tabular}

Table-7

Simulation results of an $M / M / 2 / N$ queueing model with two heterogeneous servers subject to modified

binomially distributed catastrophic intensity.

Effect of change in mean service time of server $2\left(1 / \mu_{2}\right)$

Mean inter arrival time $=2$ minutes, Mean service time of

server $1=5$ minutes, mean inter catastrophe time $=100$ minutes, simulation length $=\mathbf{4 8 0}, \mathrm{N}=5$

\begin{tabular}{|c|c|c|c|c|}
$\begin{array}{c}\text { Mean } \\
\text { service } \\
\text { time of } \\
\text { server 2 }\end{array}$ & $\begin{array}{c}\text { Average } \\
\text { delay in } \\
\text { queue }\end{array}$ & $\begin{array}{c}\text { Average } \\
\text { No. in } \\
\text { queue }\end{array}$ & $\begin{array}{c}\text { Server 1 } \\
\text { Utilization }\end{array}$ & $\begin{array}{c}\text { Server 2 } \\
\text { Utilization }\end{array}$ \\
\hline
\end{tabular}

Using the property $\lim _{s \rightarrow 0} s P_{n}^{*}(s)=P_{n}$, We have from (19) to (23),

\begin{tabular}{|c|c|c|c|c|}
\hline 1 & 0.6727 & 0.0640 & 0.0599 & 0.0438 \\
\hline 2 & 1.5360 & 0.5795 & 0.6849 & 0.5215 \\
\hline 3 & 4.0723 & 0.2473 & 0.1597 & 0.1395 \\
\hline 4 & 5.2788 & 0.5434 & 0.2217 & 0.2210 \\
\hline 5 & 7.3563 & 0.8923 & 0.3362 & 0.3395 \\
\hline 6 & 7.6520 & 1.0865 & 0.3643 & 0.3662 \\
\hline 7 & 8.1255 & 1.3601 & 0.4388 & 0.4436 \\
\hline 8 & 12.1733 & 3.6002 & 0.9382 & 0.9542 \\
\hline 9 & 10.1551 & 3.1477 & 0.9384 & 0.9256 \\
\hline 10 & 8.6107 & 0.8195 & 0.2621 & 0.2627 \\
\hline
\end{tabular}

Table-8

Simulation results of an $M / M / 2 / N$ queueing model with two heterogeneous servers subject to modified binomially distributed catastrophic intensity.

Effect of change in mean inter catastrophic time $(1 / \xi)$

Mean inter arrival time $=\mathbf{2}$ minutes, Mean service time of

server $1=6$ minutes, mean service time of server $2=8$ minutes, simulation length $=\mathbf{4 8 0}, \mathrm{N}=5$

\begin{tabular}{|l|l|l|l|l|}
\hline $\begin{array}{l}\text { Mean } \\
\text { inter } \\
\text { catastrop } \\
\text { hic time }\end{array}$ & $\begin{array}{l}\text { Average } \\
\text { delay in } \\
\text { queue }\end{array}$ & $\begin{array}{l}\text { Averag } \\
\text { e No. } \\
\text { in } \\
\text { queue }\end{array}$ & $\begin{array}{l}\text { Server 1tilizatio } \\
\mathrm{n}\end{array}$ & $\begin{array}{l}\text { Server 2 } \\
\text { Utilizatio } \\
\mathrm{n}\end{array}$ \\
\hline 101 & 12.8427 & 3.6938 & 0.9433 & 0.9434 \\
\hline 102 & 9.7839 & 0.1349 & 0.0783 & 0.0781 \\
\hline 103 & 12.0068 & 2.3648 & 0.6054 & 0.6008 \\
\hline 104 & 9.6638 & 1.0964 & 0.3287 & 0.3294 \\
\hline 105 & 10.5273 & 1.5314 & 0.4582 & 0.4469 \\
\hline 106 & 10.3545 & 1.1666 & 0.3428 & 0.3408 \\
\hline 107 & 10.9170 & 1.1578 & 0.3221 & 0.3248 \\
\hline 108 & 10.1586 & 1.9845 & 0.5836 & 0.5662 \\
\hline 109 & 10.5369 & 0.9082 & 0.2831 & 0.2846 \\
\hline 110 & 12.1190 & 1.1724 & 0.3512 & 0.3458 \\
\hline
\end{tabular}

\section{Steady State Solution}




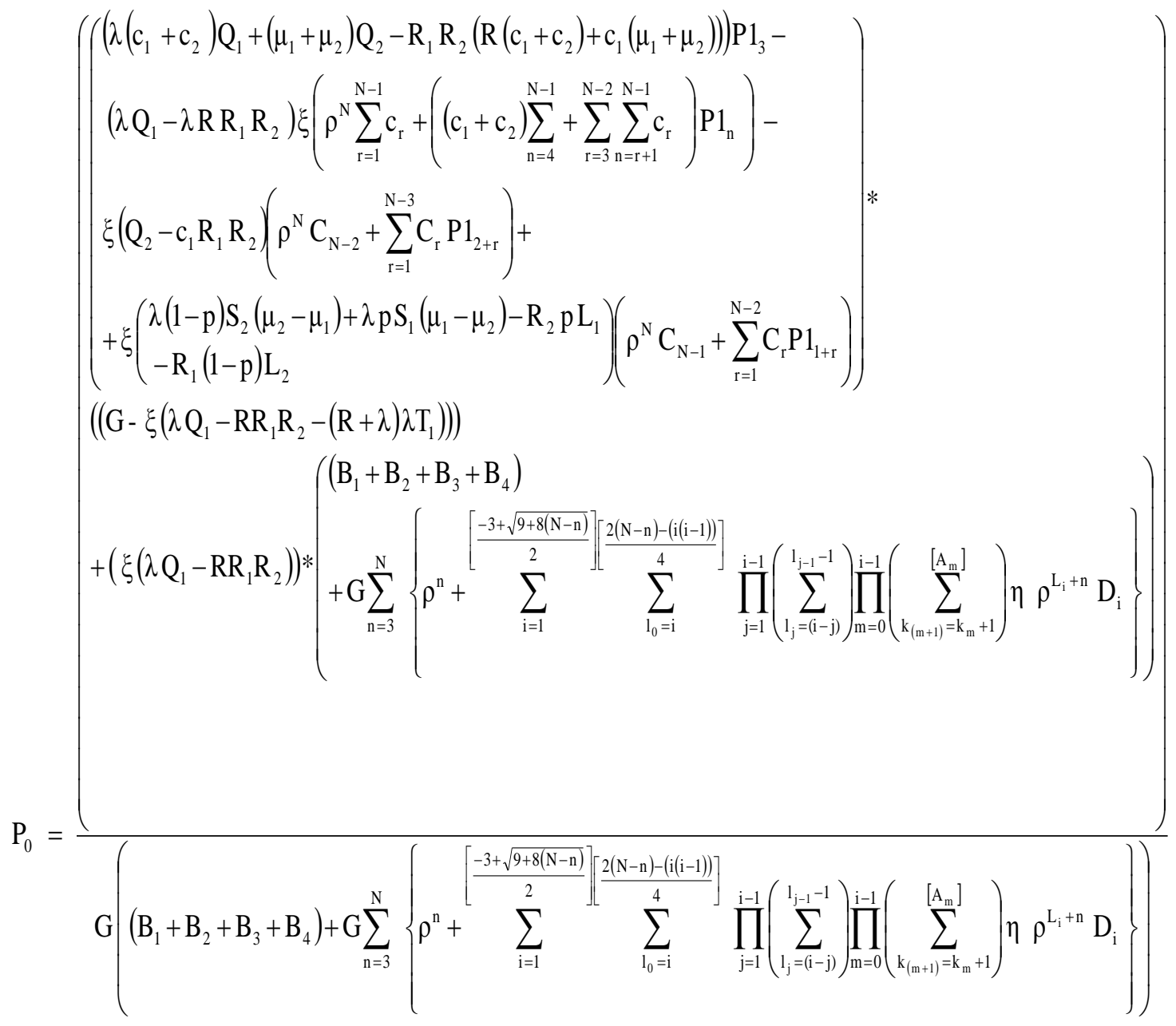




$$
\begin{aligned}
& \left(\left(\left(-\left(\lambda^{2} \xi\left(\mathrm{c}_{1}+\mathrm{c}_{2}\right)+\lambda \mu_{2}\left(\mu_{1}+\mu_{2}\right)\right)\right) \mathrm{U}_{1}+\mathrm{R}_{2}\left(\begin{array}{l}
\lambda \mathrm{p}\left(\mathrm{R}\left(\mathrm{c}_{1}+\mathrm{c}_{2}\right)+\mathrm{c}_{1}\left(\mu_{1}+\mu_{2}\right)\right) \\
-(\lambda+\xi)\left(\mu_{1}+\mu_{2}\right) \mathrm{S}_{2}
\end{array}\right)\right) \mathrm{P}_{3}+\right. \\
& \left(\left(-\lambda^{2} U_{1}+\lambda p R R_{2}\right) \xi\left(\rho^{N} \sum_{r=1}^{N-1} c_{r}+\left(\left(c_{1}+c_{2}\right) \sum_{n=4}^{N-1}+\sum_{r=3}^{N-2} \sum_{n=r+1}^{N-1} c_{r}\right) P 1_{n}\right)\right)+ \\
& \left(-\lambda \xi \mu_{2} \mathrm{U}_{1}+\lambda \mathrm{p} \xi \mathrm{c}_{1} \mathrm{R}_{2}-(\lambda+\xi) \xi \mathrm{R}_{2} \mathrm{~S}_{2}\right)\left(\rho^{\mathrm{N}} \mathrm{C}_{\mathrm{N}-2}+\sum_{\mathrm{r}=1}^{\mathrm{N}-3} \mathrm{C}_{\mathrm{r}} \mathrm{P} 1_{2+\mathrm{r}}\right) \\
& +\left(\lambda \xi(\lambda+\xi) \mathrm{U}_{1}-(\lambda+\xi) \xi \mathrm{pRR} \mathrm{R}_{2}\right)\left(\rho^{\mathrm{N}} \mathrm{C}_{\mathrm{N}-1}+\sum_{\mathrm{r}=1}^{\mathrm{N}-2} \mathrm{C}_{\mathrm{r}} \mathrm{P} 1_{1+\mathrm{r}}\right) \\
& \left(\left(G-\xi\left(\lambda Q_{1}-R_{1} R_{2}-(R+\lambda) \lambda T_{1}\right)\right)\right)+
\end{aligned}
$$

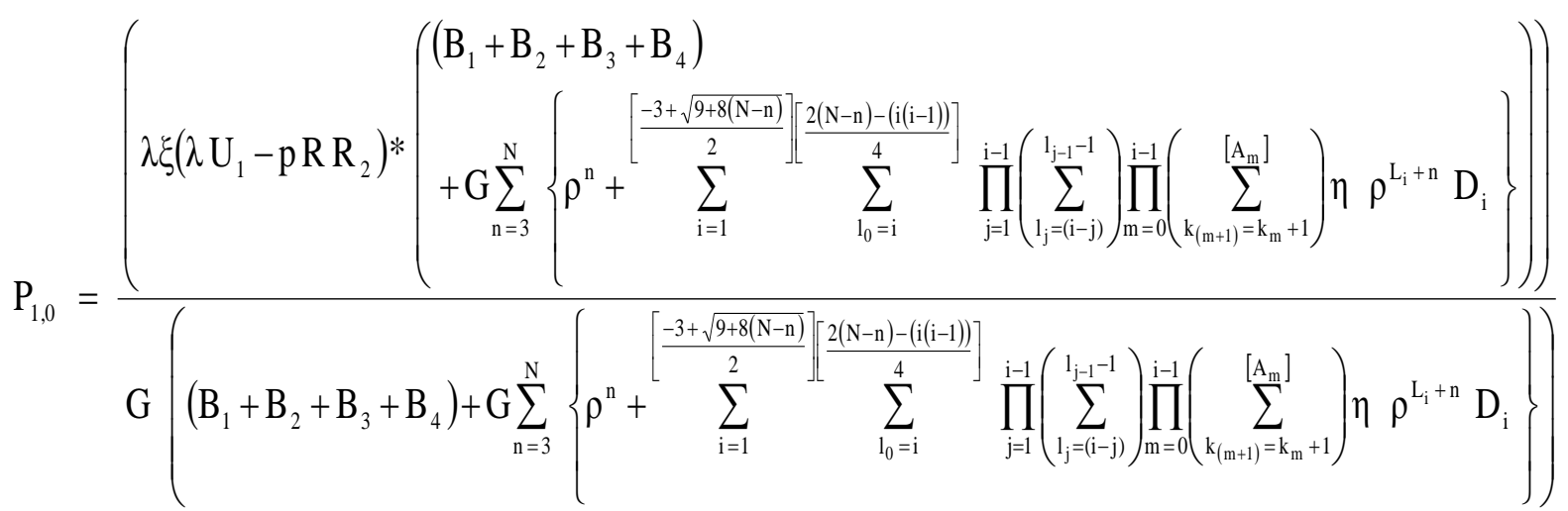

$$
\begin{aligned}
& \int\left(\left(\left(\lambda^{2} \xi\left(\mathrm{c}_{1}+\mathrm{c}_{2}\right)+\lambda \mu_{1}\left(\mu_{1}+\mu_{2}\right)\right)\right) \mathrm{U}_{1}+\mathrm{R}_{1}\left(\begin{array}{l}
\lambda(1-\mathrm{p})\left(\mathrm{R}\left(\mathrm{c}_{1}+\mathrm{c}_{2}\right)+\mathrm{c}_{1}\left(\mu_{1}+\mu_{2}\right)\right) \\
-(\lambda+\xi)\left(\mu_{1}+\mu_{2}\right) \mathrm{S}_{1}
\end{array}\right)\right) \mathrm{P}_{3}+ \\
& \left\{\left(\left(\lambda^{2} U_{1}+\lambda(1-p) R R_{1}\right) \xi\left(\rho^{N} \sum_{r=1}^{N-1} c_{r}+\left(\left(c_{1}+c_{2}\right) \sum_{n=4}^{N-1}+\sum_{r=3 n=r+1}^{N-2} \sum_{n}^{N-1} c_{r}\right) P 1_{n}\right)\right)+\right. \\
& \left(\lambda \xi \mu_{1} \mathrm{U}_{1}+\lambda(1-\mathrm{p}) \xi \mathrm{c}_{1} \mathrm{R}_{1}-(\lambda+\xi) \xi \mathrm{R}_{1} \mathrm{~S}_{1}\right)\left(\rho^{\mathrm{N}} \mathrm{C}_{\mathrm{N}-2}+\sum_{\mathrm{r}=1}^{\mathrm{N}-3} \mathrm{C}_{\mathrm{r}} \mathrm{P}_{2+\mathrm{r}}\right) \\
& +\left(-\lambda \xi(\lambda+\xi) \mathrm{U}_{1}-(\lambda+\xi) \xi(1-\mathrm{p}) \mathrm{R} \mathrm{R}_{1}\right)\left(\rho^{\mathrm{N}} \mathrm{C}_{\mathrm{N}-1}+\sum_{\mathrm{r}=1}^{\mathrm{N}-2} \mathrm{C}_{\mathrm{r}} \mathrm{P} 1_{1+\mathrm{r}}\right) \\
& \left(\left(G-\xi\left(\lambda Q_{1}-R R_{1} R_{2}-(R+\lambda) \lambda T_{1}\right)\right)\right)-
\end{aligned}
$$

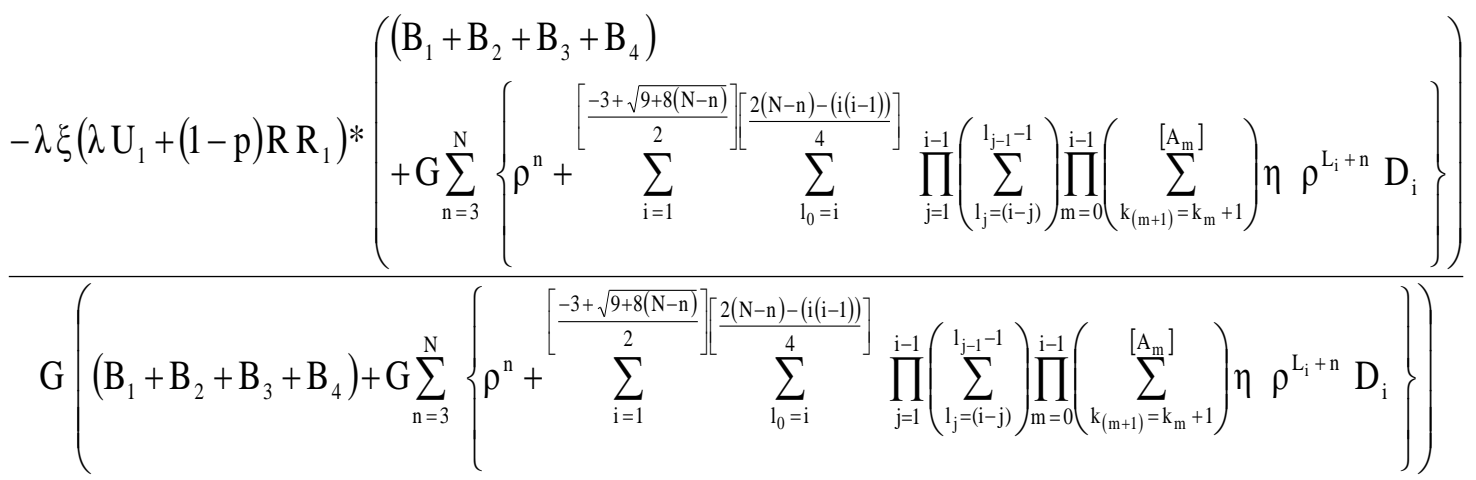




$$
\begin{aligned}
& \left\{\begin{array}{l}
\left(\begin{array}{l}
\left.\lambda^{2} \xi\left(\mathrm{c}_{1}+\mathrm{c}_{2}\right) \mathrm{T}_{1}+\lambda\left(\mu_{1}+\mu_{2}\right) \mathrm{T}_{2}-(\lambda+\xi)\left(\mu_{1}+\mu_{2}\right) \mathrm{R}_{1} \mathrm{R}_{2}\right) \mathrm{P} 1_{3} \\
+\lambda^{2} \mathrm{~T}_{1} \xi\left(\rho^{\mathrm{N}} \sum_{\mathrm{r}=1}^{\mathrm{N}-1} \mathrm{c}_{\mathrm{r}}+\left(\left(\mathrm{c}_{1}+\mathrm{c}_{2}\right) \sum_{\mathrm{n}=4}^{\mathrm{N}-1}+\sum_{\mathrm{r}=3 \mathrm{n}=\mathrm{r}+1}^{\mathrm{N}-2} \sum_{\mathrm{r}}^{\mathrm{N}-1} \mathrm{c}_{\mathrm{r}}\right) \mathrm{P} 1_{\mathrm{n}}\right)+ \\
\xi\left(\lambda \mathrm{T}_{2}-(\lambda+\xi) \mathrm{R}_{1} \mathrm{R}_{2}\right)\left(\rho^{\mathrm{N}} \mathrm{C}_{\mathrm{N}-2}+\sum_{\mathrm{r}=1}^{\mathrm{N}-3} \mathrm{C}_{\mathrm{r}} \mathrm{P} 1_{2+\mathrm{r}}\right) \\
-\lambda \xi(\lambda+\xi) \mathrm{T}_{1}\left(\rho^{\mathrm{N}} \mathrm{C}_{\mathrm{N}-1}+\sum_{\mathrm{r}=1}^{\mathrm{N}-2} \mathrm{C}_{\mathrm{r}} \mathrm{P} 1_{1+\mathrm{r}}\right)
\end{array}\right.
\end{array}\right\} * \\
& \left(\left(\mathrm{G}-\xi\left(\lambda \mathrm{Q}_{1}-\mathrm{RR}_{1} \mathrm{R}_{2}-(\mathrm{R}+\lambda) \lambda \mathrm{T}_{1}\right)\right)\right)-
\end{aligned}
$$

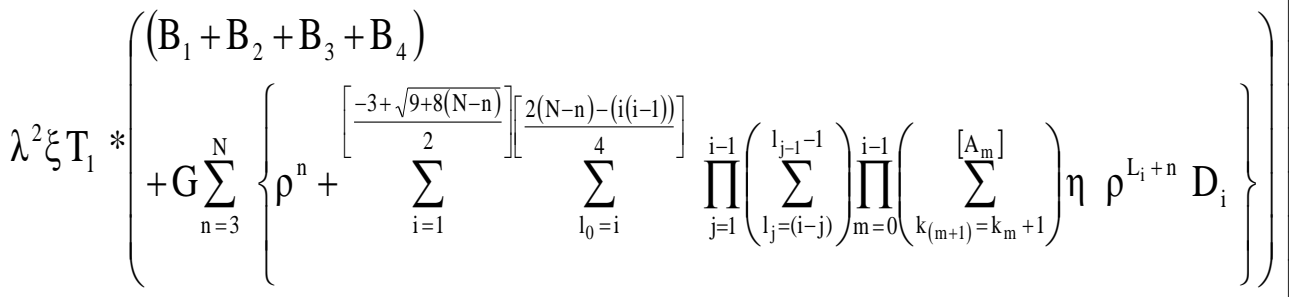

$$
\begin{aligned}
& \mathrm{P}_{2}=
\end{aligned}
$$

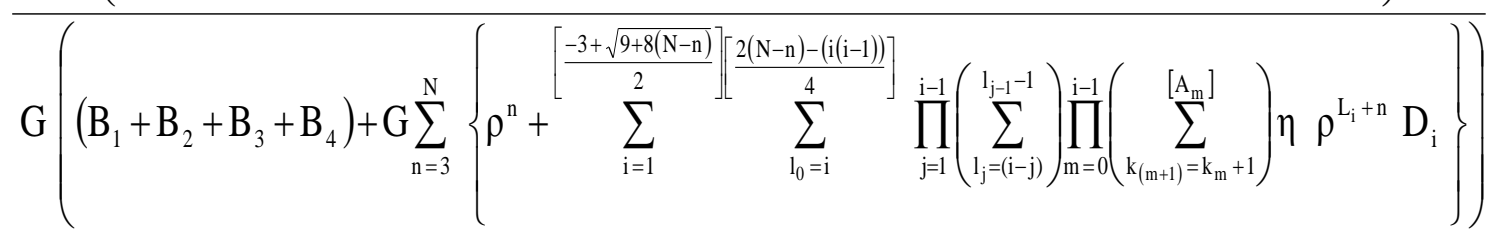

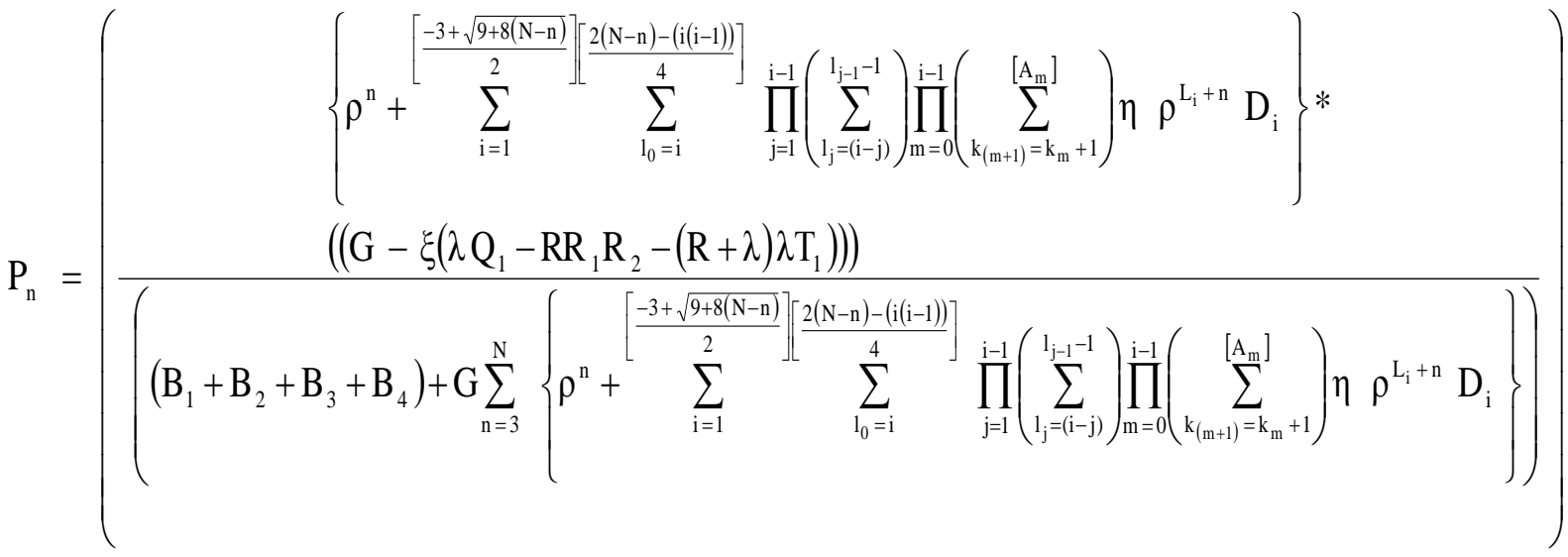

\section{CONCLUSION}

In this paper we consider two heterogeneous servers Markovian queueing system subjected to varying catastrophic intensity. The system size probabilities are calculated explicitly. The concept of varying catastrophic intensity has tremendous applications in vide variety of areas such as computer communications, agriculture and bio-sciences etc.

\section{REFERENCES}

[1] Bartoszynski, R. Buhler, W.J., Chan, W., and Pearl, D.K. (1989), Population processes under the influence of disasters occurring independently of population size, J. Math. Bio. Vol, 27, 179-190.

[2] Brockwell, P.J., Gani, J.M. and Resnick, S.I. (1982), Birth immigration and catastrophe processes, Adv. Applied Probability, Vol. 14, 709-731. 
[3] Brockwell, P.J. (1985), The extinction time of a birth, death and catastrophe process and of a related diffusion model, Advances in Applied Probability,Vol. 17, 42-52.

[4] Chao, X. (1995), A queueing network model with catastrophes and product form solution, Operations Research Letters, Vol. 18, 75-79.

[5] Chao, X. and Zheng, Y. (2003), Transient analysis of immigration birth- death processes with total catastrophes, Prob. Enggn. and Inform. Sciences, Vol. 17, 83-106.

[6] Crescenzo, A. Di, Giorno, V., Nobile, A. G. and Ricciardi, L.M. (2003), On the M/M/1 queue with catastrophes and its continuous approximation, Queueing Systems, Vol. 43, 329-347.

[7] Economou, A. (2004), The compound Poisson immigration process subject to binomial catastrophes, J. Applied Probability, Vol. 41, No. 2, 508-523.

[8] Gripenberg, G. (1983), A Stationary distribution for the growth of a population subject to random catastrophes, Journal of Mathematical Biology, Vol. 17, 371-379.

[9] Gumbel, H. (1960), Waiting lines with heterogeneous servers, Operations Research, 8, 504-511.

[10] Jain, N.K. and Kanethia, D.K. (2006), Transient analysis of a queue with environmental and Catastrophic effects, International Journal of Information and Management Sciences, Vol. 17, No.1.35-45.

[11] Jain, N.K. and Bura Gulab Singh, (2010), A queue with varying catastrophic intensity, International journal of computational and applied mathematics, Vol. 5, 41-46.

[12] Jain, N.K. and Bura Gulab Singh, (2012), two homogeneous servers limited capacity Markovian queueing system subjected to varying catastrophic intensity, International journal of computer applications, Vol. 49, No. 2, 31-41.

[13] Kitamura, K., Tokunaga, M., Hikkikoshi, I. A. and Yanagida, T. (1999), A single myosin head moves along an actin filament with regular steps of 5.3 nanometers, Nature, Vol. 397, 129-134.
[14] Kumar, B.K. and Arivudainambi, D. (2000), Transient solution of an M/M/1 queue with catastrophes, Comp. and Mathematics with Applications, Vol. 40, 1233-1240.

[15] Kumar, B.K. and Madheswari, Pavai S., (2002), Transient behavior of the $\mathrm{M} / \mathrm{M} / 2$ queue with catastrophes, Statistica, Vol. 27, 129-136.

[16] Kumar, B.K., Madheswari, Pavai S. and Venkatakrishnan, K.S., (2007), Transient solution of an $\mathrm{M} / \mathrm{M} / 2$ queue with heterogeneous servers subject to catastrophes, International Journal of Information and Management Sciences, Vol. 18, 63-80.

[17] Kyriakidis, E.G. (1994), Stationary probabilities for a simple immigration-birth-death process under the influence of total catastrophes, Stat. and Prob. Letters, Vol. 20, 239-240.

[18] Kyriakidis, E.G. (2001), the transient probabilities of the simple immigration- catastrophe process, Math.Scientist, Vol. 26, 56--58.

[19] Law, M. and Kelton, W. D., (2003), Simulation modeling and analysis, 3rd edition, McGraw Hill Book Company, New York.

[20] Liu, W. and Kumar, P., (1984), Optimal control of a queueing system with two heterogeneous servers, IEEE transactions on automatic control, Vol. 29, 696-703.

[21] Miyazaki, Y. and Sibata, S. Mori, (1992), Transient behavior of the M/M/2/ /FIFO queue with starting customers, Journal of information and optimization sciences, Vol. 13, 1-27.

[22] Narsingh Deo, (2007), System simulation with digital computer, Prentice hall of India Privat Ltd.

[23] Rubinovitch, M.,(1985), The slow server problem, Journal of applied probability, Vol. 22, 205-213.

[24] Saaty, T.L. (1960), Time dependent solution of the many server Poisson queue, Operations Research, Vol. 8, 755772 .

[25] Singh, V.P.,(1970), Two servers Markovian queues with balking: Heterogeneous vs. homogeneous servers, Operations Research, Vol. 19, 145-159. 